\title{
Properties of porous glasses with embedded ferroelectric materials
}

\author{
E. Rysiakiewicz-Pasek *, R. Poprawski, J. Polanska, A. Urbanowicz, A. Sieradzki \\ Institute of Physics, Wroclaw University of Technology, W. Wyspianskiego 27, 50-370 Wroclaw, Poland
}

Available online 14 September 2006

\begin{abstract}
Methods of introducing two ferroelectric materials, viz. $\mathrm{NaNO}_{2}$ and $\left(\mathrm{CH}_{3} \mathrm{NH}_{3}\right)_{5} \mathrm{Bi}_{2} \mathrm{Cl}_{11}(\mathrm{PMACB})$, into porous glass matrices and the results of dielectric, pyroelectric, thermal expansion and SEM investigations are presented. The best filling degree was achieved for $\mathrm{NaNO}_{2}$ introduced from the melt, whereas a significantly worse filling effect was achieved for a water solution as the introducing agent. A splitting was observed in the temperature dependence of dielectric permittivity related to the incommensurate phase of $\mathrm{NaNO}_{2}$ embedded in porous glass from a water solution, whereas no incommensurate phase was observed in the case of glass filled with $\mathrm{NaNO}_{2}$ from the melt. The confined materials turned out to possess ferroelectric properties and exhibited phase transitions. The negative size effect was observed for porous glasses filled with $\mathrm{NaNO}_{2}$ and PMACB. The above conclusions are based on the anomaly of dielectric, pyroelectric and dilatometric properties observed in the phase transition regions.
\end{abstract}

(c) 2006 Elsevier B.V. All rights reserved.

PACS: 61.43.Gt; 77.22.Ch; 77.80.Bh

Keywords: Porosity; Ferroelectric

\section{Introduction}

Ferroelectrics exhibit a number of physical features essential for their practical applications, determined by spontaneous polarization, the electric field intensity dependence of polarization, the pyro- and piezo-electric effects, electro-optical and optical properties. They are used in the production of non-volatile ferroelectric memories, piezoelectric transducers and sensors, infrared detectors, thermovision detection matrices and optoelectronic devices.

Thin ferroelectric films and low-size ferroelectric matrices are applied in such devices, so the size-effect investigation is crucial, particularly with respect to physical properties and phase transitions. The size effect is also an essential problem in fundamental research.

\footnotetext{
${ }^{*}$ Corresponding author. Tel.: +48 7132036 14; fax: +48 713283696.

E-mail address: Ewa.Rysiakiewicz-Pasek@pwr.wroc.pl (E. Rysiakiewicz-Pasek).
}

Introducing ferroelectric materials into a porous glass matrix is one of the methods of preparating nano-size ferroelectrics, but publications on porous glasses filled with a ferroelectric are few (see e.g. [1-3]). The problem seems interesting enough to be investigated further.

The aim of the present work was to develop a method of introducing two ferroelectric materials, $\mathrm{NaNO}_{2}$ and $\left(\mathrm{CH}_{3} \mathrm{NH}_{3}\right)_{5} \mathrm{Bi}_{2} \mathrm{Cl}_{11}$ (PMACB), into a porous glass matrix and to study the impact of the selected method of $\mathrm{NaNO}_{2}$ embedding on the physical properties and phase transitions of the embedded material under various conditions and with various pore sizes.

$\mathrm{NaNO}_{2}$ crystals show the ferroelectric phase transition of the order-disorder type at about $436 \mathrm{~K}$, whereupon the transition passes through an intermediate phase considered as an antiferroelectric or modulated phase. This phase appears in a $1.5 \mathrm{~K}$-wide temperature range [4] and is manifested as a splitting of the maximum in the temperature dependence of dielectric permittivity and specific heat in the phase transition regions [5]. 
PMACB is a proper ferroelectric exhibiting the secondorder phase transition of the order-disorder type at $308 \mathrm{~K}$ [6] from the $\mathrm{P}_{\mathrm{cab}}$ to $\mathrm{P}_{\mathrm{ca} 2}$ space group of the orthorombic system.

\section{Experiment}

Porous glass was fabricated by leaching sodium borosilicate glass $\left(62.6 \mathrm{wt} \%\right.$ of $\mathrm{SiO}_{2}, 30.4 \mathrm{wt} \%$ of $\mathrm{B}_{2} \mathrm{O}_{3}, 7.0 \mathrm{wt} \%$ of $\mathrm{Na}_{2} \mathrm{O}$ ) with phase separation. The original glass was subjected to heat treatment at $763 \mathrm{~K}$ for $165 \mathrm{~h}$ (in the case of glass PG1) and $933 \mathrm{~K}$ for $100 \mathrm{~h}$ (in the case of glass PG2). After leaching in hydrochloric acid, the glass samples were etched in a $\mathrm{KOH}$ solution [7]. The obtained porous glasses contained about $90 \%$ of $\mathrm{SiO}_{2}$; their basic parameters relevant to the present article are given in Table 1. Our earlier investigations indicated the presence of ultrapores in PG2's silica matrix created after the chemical and temperature treatment of the original glass [8].

Rectangular porous glass plates sized of $10 \times 10 \times$ $0.5 \mathrm{~mm}^{3}$ were filled with $\mathrm{NaNO}_{2}$ or MAPCB.

$\mathrm{NaNO}_{2}$ was introduced both from the melt and from a water solution.

The former method involved a bath of porous glass bars in melted $\mathrm{NaNO}_{2}$ at $578 \mathrm{~K}$ lasting for 4 or $16 \mathrm{~h}$. The samples were then removed from the melt and cooled in air at room temperature. The samples' surfaces were polished mechanically with sandpaper (1200) covered by a thin film of saturated water solution of $\mathrm{NaNO}_{2}$ in order to remove small $\mathrm{NaNO}_{2}$ crystals.

The latter method involved a $9 \mathrm{~h}$ bath of porous glass bars in a saturated water solution of $\mathrm{NaNO}_{2}$ at $353 \mathrm{~K}$. After removal from the solution, the samples were heated at $393 \mathrm{~K}$ for one hour to remove the absorbed water. FTIR spectra obtained for these glasses after annealing show an absence of physical water adsorption bands [work in progress].

The process of PMACB embedding in porous glass was similar to that for $\mathrm{NaNO}_{2}$ introduced from the water solution. However, it should remembered that a saturated PMACB solution is a strong acid and may thus intensify the process of ultrapores creation in PG2.

In order to perform dielectric and pyroelectric measurements, silver-paint electrodes were deposited on the large surfaces of the porous plates filled with PMACB, immediately following which they were dried.

In the case of samples filled with $\mathrm{NaNO}_{2}$ platinum electrodes are recommended due to the chemical reaction of

Table 1

Parameters of the porous glass texture

\begin{tabular}{lcc}
\hline Parameter & Glass PG1 & Glass PG2 \\
\hline Specific surface area, in $\mathrm{m}^{2} / \mathrm{g}$ & 28.9 & 5.9 \\
Pore volume, in $\mathrm{cm}^{3} / \mathrm{g}$ & 0.440 & 0.470 \\
Average diameter, in $\mathrm{nm}$ & 45 & 320 \\
Porosity, in $\%$ & 50 & 48 \\
\hline
\end{tabular}

sodium nitrite with silver and the possible diffusion of silver from the electrodes to the glass. Therefore, electrodes in the form of polished platinum plates were used in the measurements of porous glass containing $\mathrm{NaNO}_{2}$.

Dielectric permittivity measurements were performed with an Andeen Hagerling ultra-precision capacitance bridge at a frequency of $1 \mathrm{kHz}$. The frequency and temperature dependences of dielectric permittivity were studied with a Novocontrol Alpha impedance analyzer. Spontaneous polarization change measurements were performed by integrating the electric charge generated on a sample's surfaces during a given temperature change. Prior to pyroelectric measurements, the samples were polarized for $12 \mathrm{~h}$ at room temperature by an external electric field of the intensity of $4 \times 10^{5} \mathrm{~V} / \mathrm{m}$. Pyroelectric measurements were performed during the heating process of the $1 \mathrm{~K} / \mathrm{min}$ ratio. Thermal deformation was measured with a precision quartz dilatometer made in our laboratory. Dielectric and dilatometric measurements were carried out in a very slow cooling process. During the dielectric, pyroelectric and dilatometric measurements the temperature was controlled by a PID temperature controller and the results were collected by a PC.

\section{Results and discussion}

Figs. 1-4 show SEM micrographs of cleaved samples: pure PG2 glass, PG2 glass filled with $\mathrm{NaNO}_{2}$ from a water solution, $\mathrm{PG} 2$ glass filled with $\mathrm{NaNO}_{2}$ from the melt, and PG2 glass filled with PMACB, respectively. The best filling degree has been achieved for $\mathrm{NaNO}_{2}$ introduced from the melt, and the worst for PMACB. Similar conclusions can be drawn from SEM studies of PG1 glass filled with $\mathrm{NaNO}_{2}$ and PMACB.

The temperature dependence of dielectric permittivity of $\mathrm{NaNO}_{2}$ grown from the melt and porous glasses filled with $\mathrm{NaNO}_{2}$ is illustrated in Figs. 5-8. It follows from Fig. 6, which illustrates the temperature dependence of dielectric permittivity for $\mathrm{NaNO}_{2}$ embedded into PG2 glass, that

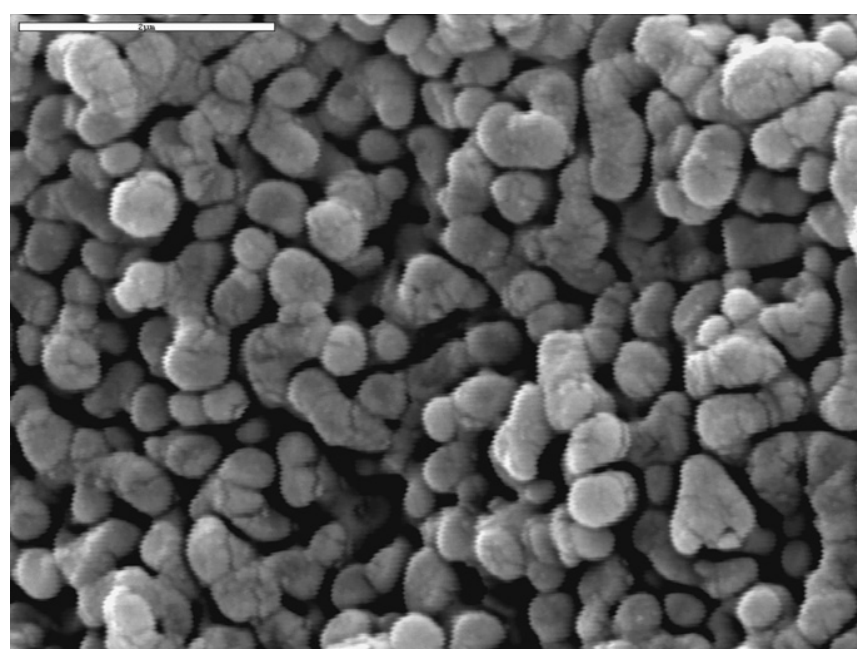

Fig. 1. SEM graph for PG2. 


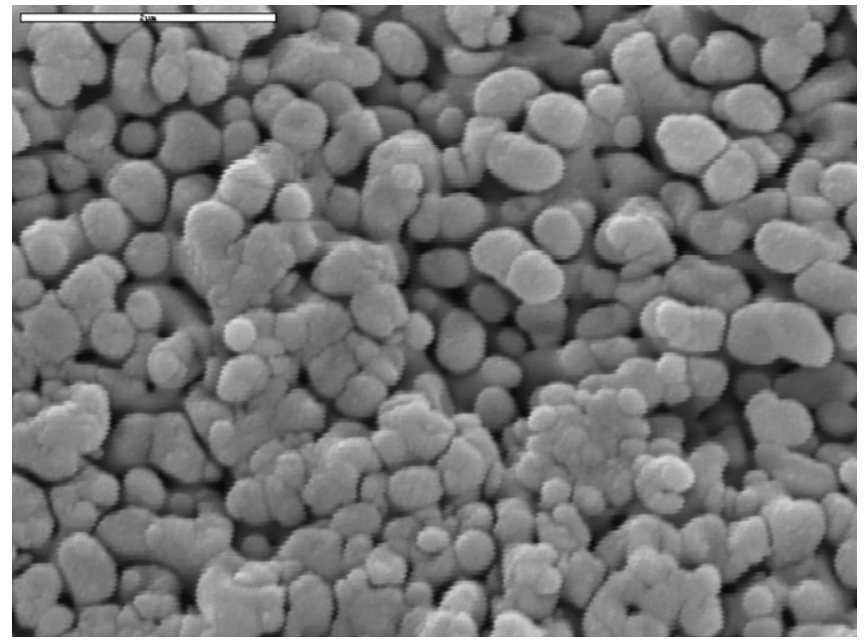

Fig. 2. SEM graph for PG2 with $\mathrm{NaNO}_{2}$ introduced from a water solution.

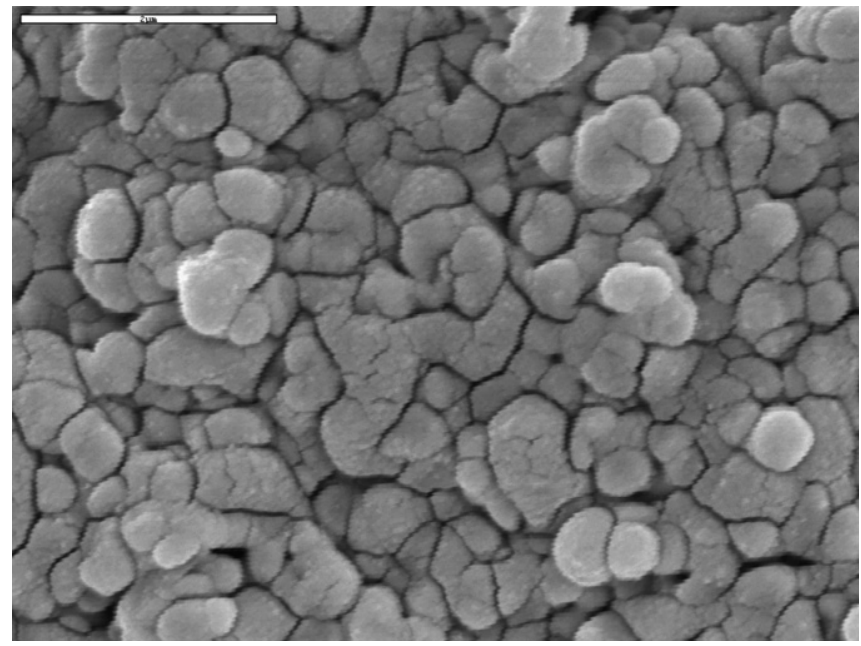

Fig. 3. SEM graph for PG2 with $\mathrm{NaNO}_{2}$ introduced from the melt.

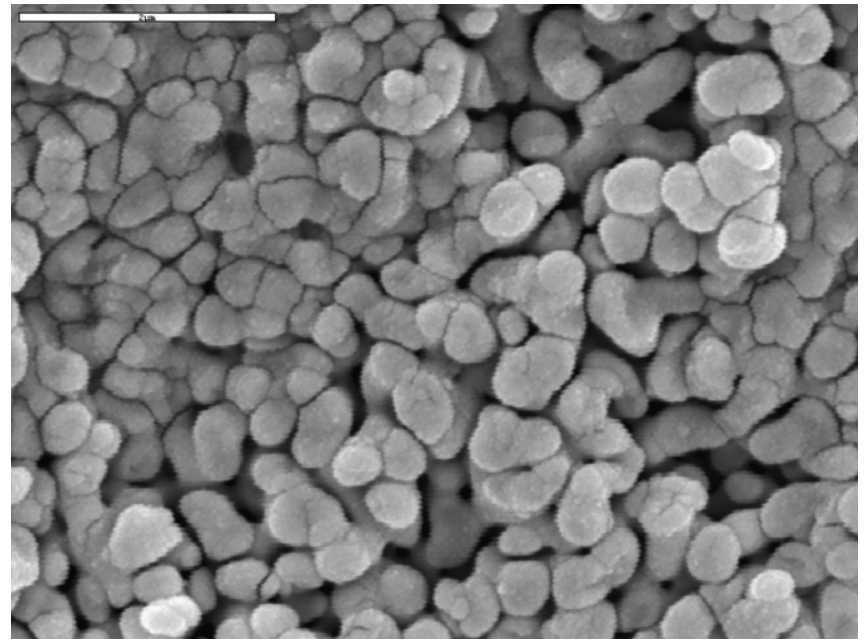

Fig. 4. SEM graph for PG2 with PMACB introduced from a water solution.

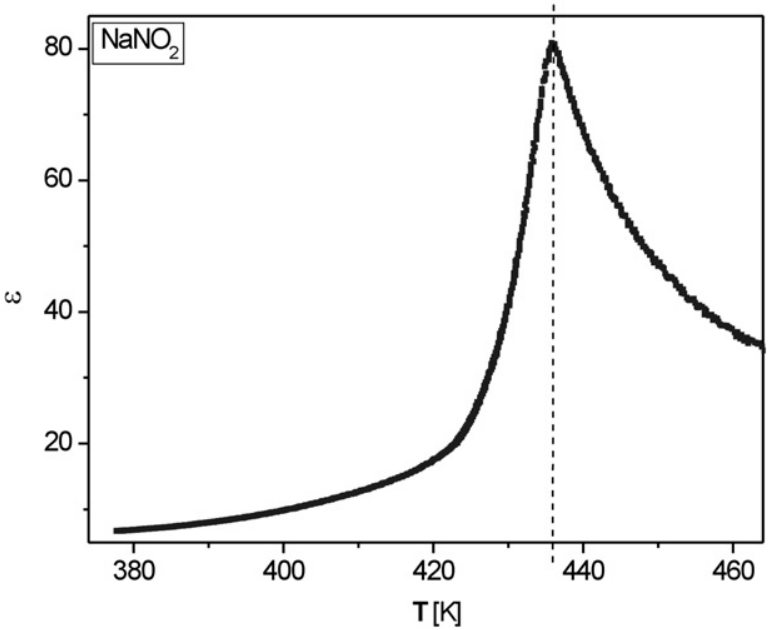

Fig. 5. Temperature dependence of dielectric permittivity, $\varepsilon$, for polycrystalline $\mathrm{NaNO}_{2}$ obtained from the melt.

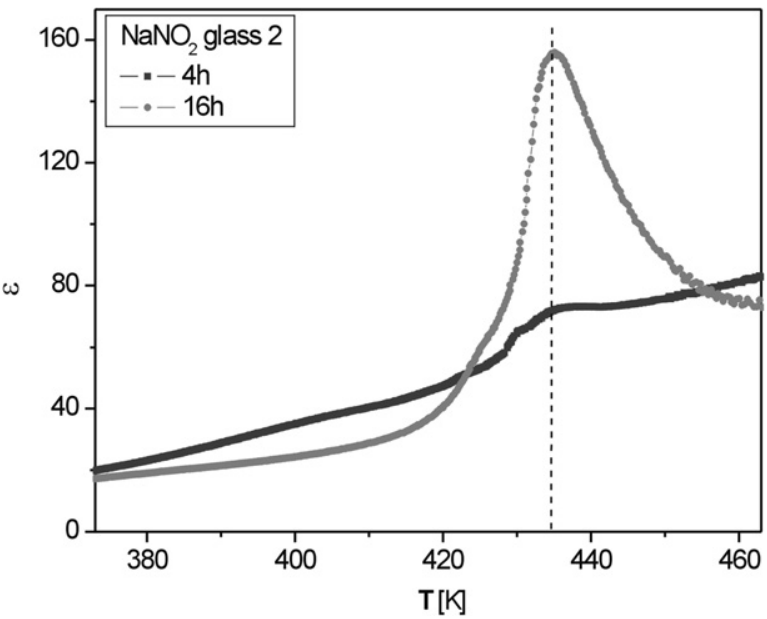

Fig. 6. Temperature dependence of dielectric permittivity, $\varepsilon$, for PG2 with $\mathrm{NaNO}_{2}$ introduced from the melt for various filling times.

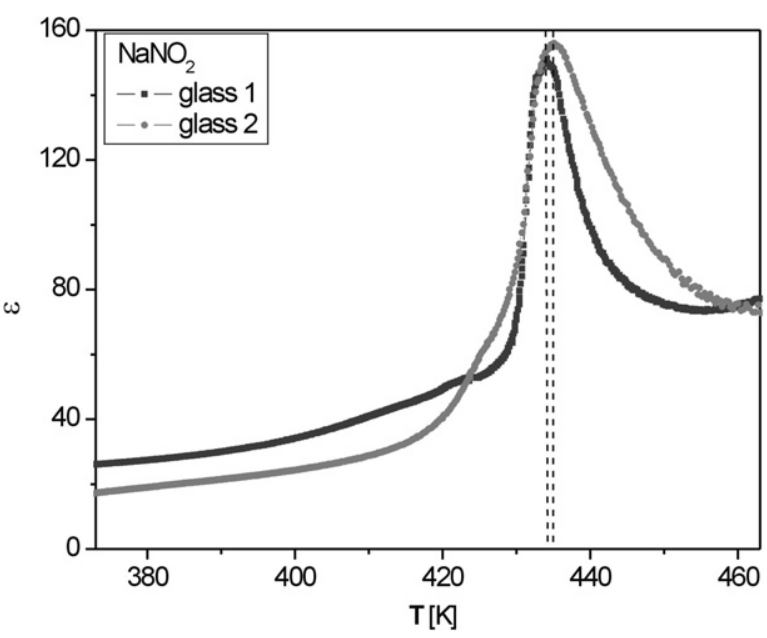

Fig. 7. Temperature dependence of dielectric permittivity, $\varepsilon$, for PG1 and PG2 2 with $\mathrm{NaNO}_{2}$ introduced from the melt. 


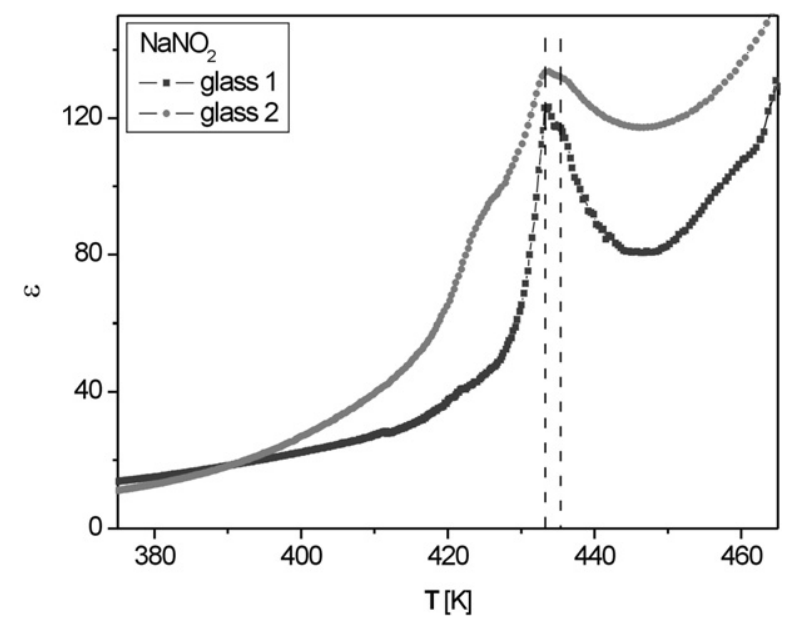

Fig. 8. Temperature dependence of dielectric permittivity, $\varepsilon$, for PG1 and PG2 with $\mathrm{NaNO}_{2}$ introduced from a water solution.

the diffusion of the melt in the glass pores is very slow. The conclusion is based on the fact that the maximum of $\varepsilon(T)$ for the 16-h bath is significantly higher than the maximum for the same glass immersed in the melt for $4 \mathrm{~h}$.

Fig. 7 shows the $\varepsilon(T)$ relation for PG1 and PG2 filled with $\mathrm{NaNO}_{2}$ by immersion in the melt for $16 \mathrm{~h}$. As the phase transition temperature decreases with the decreasing pore size, a negative size effect has been observed for $\mathrm{NaNO}_{2}$ crystals confined in the porous glass matrices. The size effect consists in a dependence of the phase transition temperature on particles' dimensions $[9,10]$. The phase transition temperatures of PG1 and PG2 filled with $\mathrm{NaNO}_{2}$ from the melt are lower than that for a bulk $\mathrm{NaNO}_{2}$ crystal. A similar conclusion can be drawn from the $\varepsilon(T)$ relation for $\mathrm{NaNO}_{2}$ introduced into porous glass from the water solution (Fig. 8).

Notably, the maximum value of dielectric permittivity of PG1 and PG2 filled with $\mathrm{NaNO}_{2}$ is about double that observed in the polycrystalline $\mathrm{NaNO}_{2}$ sample. Similar results for $\mathrm{NaNO}_{2}$ embedded in other porous materials were presented by Tien et al. [11] and Kutnjak et al. [12]. Maximum dielectric permittivity of polycrystalline $\mathrm{NaNO}_{2}$ is significantly sharper than that for porous glass samples filled with $\mathrm{NaNO}_{2}$. The maximum permittivity smearing in the case of glass filled with $\mathrm{NaNO}_{2}$ from the melt (Fig. 7) may be due to the distribution of microcrystal sizes according to various pore dimensions and/or from the electric field induced at the glass-ferroelectric interface. An external electric field in bulk $\mathrm{NaNO}_{2}$ crystals reduces dielectric permittivity around the phase transition and leads to a gradual disappearance of the intermediate phase, as referred to in the literature [5]. The absence of splitting in the maximum temperature dependence of dielectric permittivity in a polycrystalline sample grown by fast cooling from the melt indicates that the disappearance of the incommensurate phase is related to crystallites' size and their grain surface more than to an interaction between the glass matrix and ferroelectric microcrystals.
Mechanical strains due to the difference in thermal expansion coefficients of the glass matrix and the ferroelectric in its pores can influence significantly the shape and position of the maximum of dielectric permittivity. Hydrostatic pressure of $500 \mathrm{MPa}$ has caused a positive shift of the phase transition temperature of single $\mathrm{NaNO}_{2}$ crystals of about $15 \mathrm{~K}$ [5].

The splitting of the maximum of dielectric permittivity's temperature dependence is clearly visible in Fig. 8, caused by a phase transition from the intermediate to the paraelectric phase. Notably, this is the first result showing the existence of the intermediate phase in $\mathrm{NaNO}_{2}$ embedded in porous materials. As the splitting was observed in the samples prepared from a water solution, we can conclude that the physical properties of $\mathrm{NaNO}_{2}$ microcrystals obtained with this method are much more similar to those observed in bulk crystals than those of $\mathrm{NaNO}_{2}$ obtained by fast cooling of the melt.

Preliminary pyroelectric studies of porous glass filled with $\mathrm{NaNO}_{2}$ from the melt have shown sharp maxima of the pyroelectric current at the phase transition temperature, which is evidence of the glass's ferroelectric properties. The temperature dependence of the electric charge generated on the surface of the PG2 sample filled with $\mathrm{NaNO}_{2}$ (proportional to the spontaneous polarization changes) is shown in Fig. 9. The phase transition temperatures obtained in the pyroelectric and the dielectric studies are different, which may be due to either thermal hysteresis or a small shift between the temperature of the sample and that measured by the experimental system. The pyroelectric measurements were carried out while the heating rate was relatively high $(1 \mathrm{~K} / \mathrm{min})$.

Fig. 10 shows the temperature dependence of dielectric permittivity for PG2 glass filled with PMACB measured in the frequency range from $1.84 \times 10^{5}$ to $1.35 \times 10^{6} \mathrm{~Hz}$. A small but distinct maximum is proof of the ferroelectric

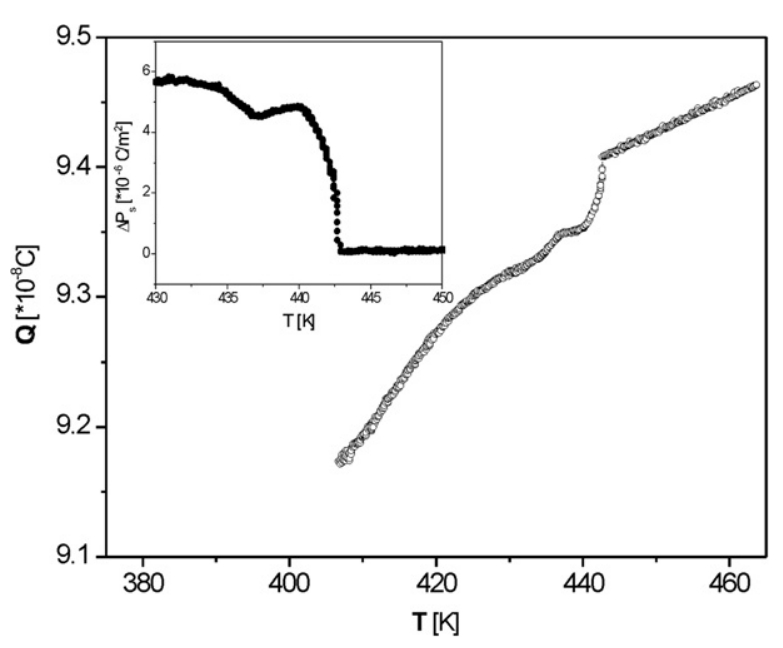

Fig. 9. Temperature dependence of the electric charge generated on the surface of $\mathrm{PG} 2$ with $\mathrm{NaNO}_{2}$ introduced from the melt during temperature changes. The spontaneous polarization changes in the phase transition region are shown in the insert as a function of temperature. 


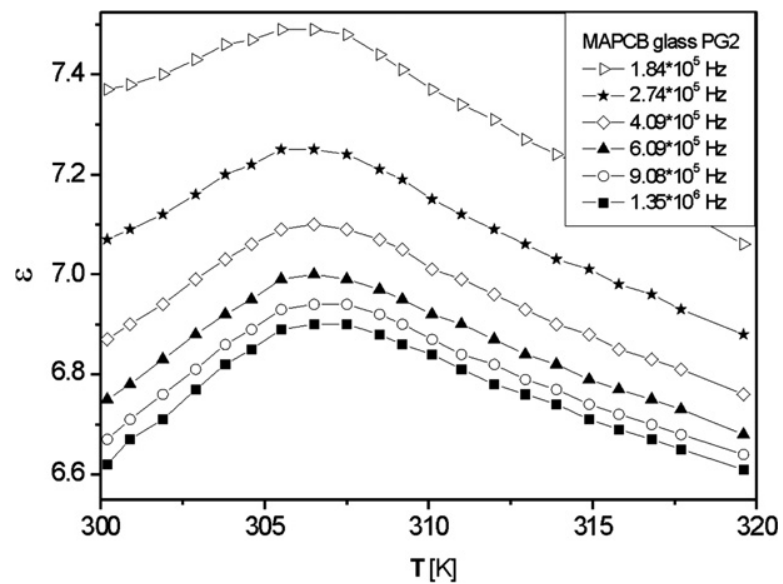

Fig. 10. Temperature dependence of dielectric permittivity, $\varepsilon$, for PG2 with PMACB, various frequencies.

phase transition occurring in the measured sample. There is a shift of the phase transition temperature towards lower values when compared to PMACB crystal. There is also a notable dispersion of dielectric permittivity for both phases, while dispersion is significantly higher in the ferroelectric phase. The presented dielectric results of porous glass filled with PMACB are limited to relatively high frequencies, as no maximum of dielectric permittivity in the phase transition region was observed at lower frequencies.

Figs. 11 and 12 show dilatometric measurement results for PG1 and PG2 glasses filled with PMACB. A continuous change of the temperature dependence of the thermal deformation and a change of its slope at the phase transition temperature for PG1 filled with PMACB are clearly visible. The abrupt change of the thermal expansion coefficient (characteristic for second-order phase transitions) at $311 \mathrm{~K}$ is presented in the insert of Fig. 11 .

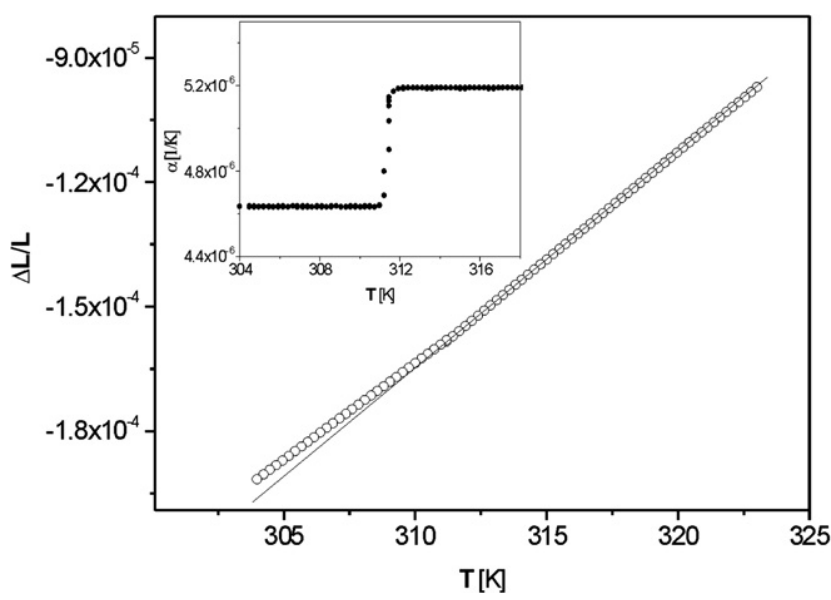

Fig. 11. The relative thermal deformation. $\Delta L / L$, and the thermal expansion coefficient, $\alpha$, (in the insert) of PG1 with PMACB as a function of temperature. The straight line denotes an approximation of the temperature dependence of thermal dilatation from the para-electric to the ferroelectric phase.

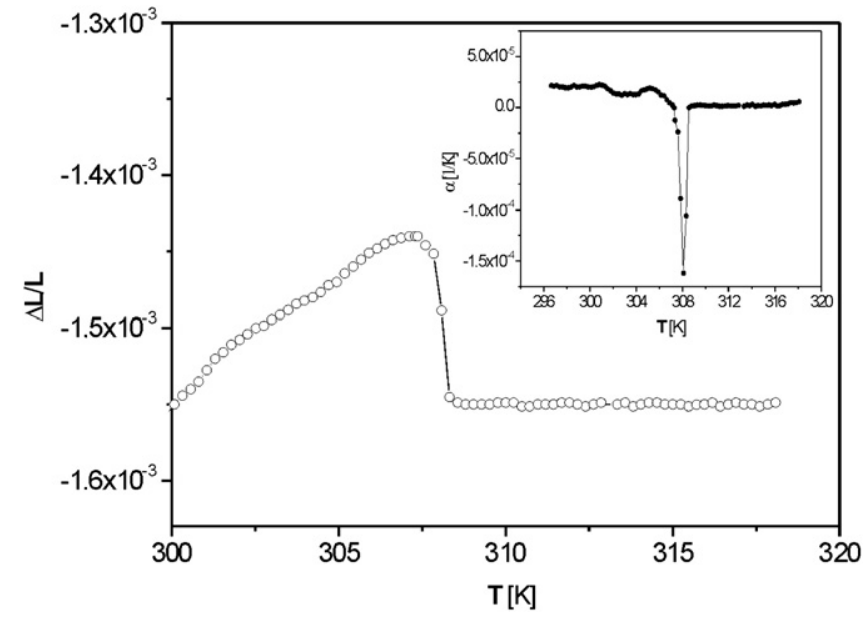

Fig. 12. Temperature dependences of the relative thermal deformation, $\Delta L / L$, and the thermal expansion coefficient $\alpha$ (in the insert) of PG2 with PMACB.

A jump of thermal deformation at the phase transition was observed in the case of PMACB embedded in PG2. This jump was probably due to the pore wall cracking during the samples' heating in the phase transition region. Ultrapores are characteristic for PG2 glass filled with PMACB. The volume expansion coefficient of PMACB embedded in the ultrapores is much higher than that for the glass matrix. The mechanical stress caused by the thermal expansion of PMACPB in the ultrapores is capable of destroying the pore walls.

\section{Conclusions}

i. The technological methods described in the present work demonstrate the feasibility of introducing $\mathrm{NaNO}_{2}$ and PMACB into porous glass matrices. The best filling degree has been achieved when introducing $\mathrm{NaNO}_{2}$ from the melt.

ii. Ferroelectric phase transitions in $\mathrm{NaNO}_{2}$ and PMACB introduced into porous glass were observed by three independent methods.

iii. The splitting of the temperature dependence of dielectric permittivity was observed in the phase transition region related to the incommensurate phase in porous glass filled with $\mathrm{NaNO}_{2}$ from a water solution. This is the first report on the splitting in $\mathrm{NaNO}_{2}$ embedded in porous glass. The intermediate phase was not observed in porous glass filled with $\mathrm{NANO}_{2}$ from the melt.

iv. A negative size effect has been observed in porous glass filled with $\mathrm{NaNO}_{2}$ and PMACB. The observed phase transition temperatures tend to decrease with the decreasing of pore sizes.

v. The texture of porous glass may be strongly affected during PMACB introduction. This phenomenon was especially important for the PG2 glass and was attributable to the existence of ultrapores in PG2 specimens. 
vi. Further complex studies are necessary to comprehend fully the process of introducing various ferroelectric materials into porous glasses and to understand the process's influence on the physical properties and phase transitions of materials embedded in porous glasses.

\section{Acknowledgement}

The present work has been financially supported by the Polish Ministry of Scientific Research and Information Technology, Department of Scientific Research (under Grant No. 3 T08 D 00726).

\section{References}

[1] E.V. Colla, A.V. Fokin, Yu.A. Kumzerov, Solid State Comm. 103 (1997) 127.

[2] B. Dorner, I. Golosovsky, Yu. Kumzerov, D. Kurdukov, A. Naberezhnov, N. Okuneva, S. Vakhrushev, in: Symposium on Ferroelecticity, St Petersburg, 2002, 191.
[3] A. Nabereznov, A. Fokin, Yu. Kumzerov, A. Sobotnikov, S. Vakhrushev, B. Dorner, Eur. Phys. J. E 12 (2003) S21.

[4] M.E. Lines, A.M. Glass, Principles and Applications of Ferroelectrics and Related Materials, Claredon, Oxford, 1977, pp. 368-373.

[5] Landolt-Bornstein, Numerical Data and Functional Relationships in Science and Technology, Group III-Crystal and Solid State Physics, vol. 16, Ferroelectrics and Related Substances, Subvolume b: Non-Oxides, Springer-Verlag, Berlin-Heidelberg-New York, 1982.

[6] R. Jakubas, L. Sobczyk, J. Lefebvre, Ferroelectrics 100 (1989) 143.

[7] E. Rysiakiewicz-Pasek, P. Lukaszewski, J. Bogdanska, Optica Applicata XXX (2000) 173.

[8] A. Gutina, T. Antropova, E. Rysiakiewicz-Pasek, K. Virnik, Yu. Feldman, Micropor. Mesopor. Mater. 58 (2003) 237.

[9] W.L. Zhong, Y.G. Wang, P.L. Zhang, B.D. Qu, Phys. Rev. B 50 (1994) 698.

[10] B.A. Strukov, S.T. Davitadze, S.G. Shulman, B.V. Goltzman, V.V. Lemanov, Ferroelectrics 301 (2004) 157.

[11] C. Tien, E.V. Charnaya, S.V. Baryshnikov, M.K. Lee, S.Y. Sun, D. Michel, W. Bohlman, Fiz. Tverd. Tela 46 (2004) 2224.

[12] Z. Kutnijak, B. Vodopivec, R. Blinc, A. Fokin, Yu. Kumzerov, S. Vakhrushev, J. Chem. Phys. 123 (2005) 084708. 